Pacific Journal of Mathematics

MAXIMAL GROUPS IN SANDWICH SEMIGROUPS OF BINARY

KeN CHAS 


\title{
MAXIMAL GROUPS IN SANDWICH SEMIGROUPS OF BINARY RELATIONS
}

\author{
KAREN ChASE
}

\begin{abstract}
A sandwich semigroup is given as follows. Let $R$ be an arbitrary but fixed binary relation on a finite set $X$. For relations $A$ and $B$ on $X$ we say $(a, b) \in A * B$ (the product of $A$ and $B$ ) if there are $c$ and $d$ in $X$ such that $(a, c) \in A$, $(c, d) \in R$ and $(d, b) \in B$. This semigroup is denoted $B_{X}(R)$. In this paper we study maximal groups in $B_{X}(R)$ for various classes of $R$.
\end{abstract}

Sandwich semigroups of binary relations were introduced in [2]. These semigroups arise naturally in automata theory, and their role in automata theory is studied in [3]. Montague and Plemmons [5] have shown that given a finite group $G$ there is some set $X$ such that $G$ is a maximal group in $B_{X}$, the usual semigroup of binary relations. We show there are classes of $R$ for which this result holds and others for which it does not hold.

If $R$ is a relation and $E$ is a nonzero idempotent in $B_{X}(R)$, then we write $G_{E}(R)$ for the maximal group determined by $E$ and call $E$ an $R$-idempotent. In $\S 1$ we give a class of relations for which $G_{E}(R)$ is trivial for any relation $R$ in this class and any $R$-idempotent $E$. In $\S 2$ we produce a class of relations for which the MontaguePlemmons result holds. That is, any finite group $G$ arises as a maximal group for some $X$ and some relation $R$ in this class. Finally, in $\S 3$ we show there is a class of relations for which some but not all finite groups arise.

Throughout we use Boolean matrix representation for relations. That is, if $R$ is a relation over $X$ where $|X|=n$, then $R$ is represented by an $n \times n$ matrix where the $(i, j)$ entry is a 1 if $\left(x_{i}, x_{j}\right)$ is in $R$ and 0 otherwise. These matrices are multiplied using Boolean arithmetic.

This paper is part of a $\mathrm{Ph} . \mathrm{D}$. thesis prepared under the direction of C. J. Maxson whom I wish to thank for his guidance and many helpful suggestions.

1. $B_{X}(R)$ containing only trivial groups. Let $\Gamma$ be the collection of (nonzero) matrices with the property that all nonzero columns are the same. For $R$ in $\Gamma$ it is easy to see that if the $(i, j)$ entry of $R$ is zero then either row $i$ or column $j$ of $R$ is zero. The following theorem characterizes $R$-idempotents for any $R$ in $\Gamma$ and shows that $G_{E}(R)$ is trivial for any $R$ in $\Gamma$ and any $R$-idempotent $E$. 
THEOREM 1. Let $R$ be in $\Gamma$. Then

(i) $A$ is an $R$-idempotent if and only if all nonzero rows of $A$ are the same and for some $i$ and $j$ such that the $(i, j)$ entry of $R$ is nonzero we have the $(j, i)$ entry of $A$ is nonzero.

(ii) If $E$ is an $R$-idempotent, then $G_{E}(R)$ is trivial.

Proof. Throughout the proof let $a_{i j}\left(r_{i j}\right)$ denote the $(i, j)$ entry of the matrix $A(R)$.

(i) Assume $A$ is an $R$-idempotent. $A R$ has zero columns where $R$ does and since all nonzero columns of $R$ are alike, all nonzero columns of $A R$ are alike. Let

$$
\left(\begin{array}{c}
b_{1} \\
b_{2} \\
\vdots \\
b_{n}
\end{array}\right)
$$

denote the nonzero columns of $A R$. Writing out the product $A R A$ we see that for each $i$ such that $b_{i}=1$ we have a nonzero row of $A$ and each nonzero row is identical.

Assume for each $k$ and $m$ such that $r_{k m}=1$ we have $a_{m k}=0$. Clearly, if column $j$ of $R$ is zero, then column $j$ of $A R$ is zero. We show if column $j$ of $R$ is nonzero, then row $j$ of $A$ is zero. These two statements imply $(A R) A=0$, a contradiction. Let column $j$ of $R$ be nonzero and denote by $b_{j i}$ the $(j, i)$ entry of $A R$. Then for any $i$

$$
\begin{aligned}
b_{j i}=\sum_{k=1}^{n} a_{j k} r_{k i} & = \begin{cases}\sum_{k=1}^{n} a_{j k} r_{k j} & \text { if column } i \text { of } R \text { is nonzero } \\
0 & \text { (hence } \left.r_{k i}=r_{k j}\right)\end{cases} \\
& =0 \text { in either case by the assumption. }
\end{aligned}
$$

Thus row $j$ of $A R$ is zero which implies row $j$ of $(A R) A=A$ is zero.

Conversely, assume $r_{i j}=1$ and $a_{j i}=1$. If row $k$ of $A$ is nonzero, then $a_{k i}=1$. From $a_{k i}=r_{i j}=a_{j i}=1$ we have the $(k, i)$ entry of $A R A$ is 1 and so row $k$ of $A R A$ is nonzero. Since $a_{k i}=1$, row $k$ of $A R$ is row $i$ of $R$ and so the $(k, j)$ entry of $A R$ is nonzero. Furthermore, since $a_{j i}=1$ we have row $k$ of $A R A$ is row $j$ of $A$. But all rows of $A$ are the same so row $k$ of $A R A$ is row $k$ of $A$. If row $k$ of $A$ is zero, then row $k$ of $A R A$ is zero. Hence we have $A R A=A$ and $A$ is an $R$-idempotent.

(ii) Let $E$ be an $R$-idempotent and $A$ be in $G_{E}(R)$. Throughout the remainder of the proof we use the following: 
$e_{i j}$ denotes the $(i, j)$ entry of $E$,

$b_{i j}$ denotes the $(i, j)$ entry of $A R$,

$c_{i j}$ denotes the $(i, j)$ entry of $A R E$.

We show $a_{i j}=e_{i j}$ for any $i$ and $j$.

Let $e_{i j}=0$. Then, by the remark preceding the theorem, either row $i$ or column $j$ of $E$ is zero. If row $i$ is zero, then row $i$ of $E R A=A$ is zero and so $a_{i j}=0$. If column $j$ is zero, then column $j$ of $A R E=A$ is zero and so $a_{i j}=0$.

Let $e_{i j}=1$. We show $a_{i j}=1$. Assume not, that is assume $a_{i j}=0$. We first show row $i$ and column $j$ of $A$ are zero. We have

$$
c_{i j}=\sum_{k=1}^{n} b_{i k} e_{k j} .
$$

Since all nonzero columns of $E$ are alike, then for any nonzero columns $n$ and $j$ of $E$ it follows that $c_{i j}=c_{i m}$. But $A R E=A \mathrm{im}$ plies $c_{i j}=a_{i j}=0$ and so row $i$ of $A$ is zero. Similarly column $j$ of $A$ is zero.

We now show $A=0$, a contradiction. If row $k$ of $E$ is zero, then $E R A=A$ implies row $k$ of $A$ is zero. If row $k$ of $E$ is nonzero, then $e_{k j}=1$ since $e_{i j}=1$. By the above we know column $j$ of $A$ is zero, so $a_{k j}=0$. Thus we have $e_{k j}=1$ and $a_{k j}=0$. Using the above arguments, this implies row $k$ of $A$ is zero.

2. $B_{X}(R)$ containing all finite groups. Let $\Gamma$ be any class of matrices such that for every positive integer $n$ the matrix

$$
\left(\begin{array}{ll}
I_{n} & A \\
B & C
\end{array}\right)
$$

is in $\Gamma$ where $I_{n}$ is the $n \times n$ identity matrix, $A$ is an arbitrary $n \times k$ matrix, $B$ is an arbitrary $k \times n$ matrix and $C$ is an arbitrary $k \times k$ matrix.

THEOREM 2. If $G$ is a finite group, then $G$ is a maximal group in $B_{X}(R)$ for some nonidentity matrix $R$ in $\Gamma$ and some $X$.

Proof. From Montague and Plemmons [5] we know there is an $X^{\prime}$ such that $G$ is isomorphic to $G_{E^{\prime}}(I)$ where $E^{\prime}$ is an idempotent in $B_{X^{\prime}}(I)$ ( $I$ is the identity relation). Let $X^{\prime}$ have $n$ elements and

$$
R=\left(\begin{array}{ll}
I_{n} & A \\
B & C
\end{array}\right)
$$

where $R$ is $k \times k$ with $k$ greater than $n$ and $A, B$ and $C$ are arbitrary. The matrix $E$ where 


$$
E=\left(\begin{array}{ll}
E^{\prime} & 0 \\
0 & 0
\end{array}\right)
$$

is an $R$-idempotent. Let $A$ be in $G_{E}(R)$ where

$$
A=\left(\begin{array}{ll}
P & Q \\
R & S
\end{array}\right) \text {. }
$$

Then, $A * E=A=E * A$ gives $Q=R=S=0$ and $P E^{\prime}=E^{\prime} P=P$. Let $B$ be the $R$-inverse of $A$ in $G_{E}(R)$. Then

$$
B=\left(\begin{array}{ll}
P^{\prime} & 0 \\
0 & 0
\end{array}\right)
$$

and $B * A=E=A * B$ give $P P^{\prime}=E=P^{\prime} P$ and so $P$ is in $G_{E^{\prime}}$. Thus the map $\theta$ from $G_{E^{\prime}}(I)$ to $G_{E}(R)$ given by

$$
\theta(P)=\left(\begin{array}{ll}
P & 0 \\
0 & 0
\end{array}\right)
$$

is an isomorphism.

We remark here that the $R$ and $X$ of the theorem are not unique. In fact $G$ is in $B_{X}(R)$ for all $X$ containing at least $n$ elements. Also, if $R$ is as in the theorem and $R^{\prime}=P R Q$ where $P$ and $Q$ are invertible, then the map $\theta$ from $B_{X}(R)$ onto $B_{X}\left(R^{\prime}\right)$ given by $\theta(A)=Q A P$ is an isomorphism.

The following theorem shows the symmetric groups arise in $B_{X}(R)$ where $R$ is a permutation.

THEOREM 3. Let $R$ be a permutation in $B_{X}(I)$ for some arbitrary but fixed $X$ where $X$ has $n$ elements. Then $R^{\prime}$, the inverse of $R$ in $B_{X}(I)$, is an $R$-idempotent and $G_{R^{\prime}}(R)$ is isomorphic to $S_{n}$, the symmetric group on $n$ elements.

Proof. It is clear that $R^{\prime}$ is an $R$-idempotent, and for all $A$ in $B_{X}(R)$ we have $A * R^{\prime}=R^{\prime} * A=A$. It remains to be shown that only permutations have an $R$-inverse with respect to $R^{\prime}$. If $A$ is a permutation, then $A R$ and $R A$ are permutations and $\left(R^{\prime} A^{\prime} R^{\prime}\right)(R A)=$ $(A R)\left(R^{\prime} A^{\prime} R^{\prime}\right)=R^{\prime}$ where $A^{\prime}$ is the $I$-inverse of $A$. Thus, $R^{\prime} A^{\prime} R^{\prime}$ is the $R$-inverse of $A^{\prime}$.

Conversely, assume for some $A$ we have a $B$ such that $A * B=$ $B * A=R^{\prime}$. If $A$ is not a permutation, then either $x A=\varnothing$ for some $x$ in $X$ or for some $x$ and $y$ in $X$ with $x \neq y$ we have $x A=y A$. In the former case we have $\varnothing=x(A * B)=x R^{\prime}$. In the latter case since $R$ is a permutation, we have $x(A * B)=y(A * B)$ and so $x\left(R^{\prime}\right)=$ $y\left(R^{\prime}\right)$ for $x \neq y$. Neither case is tenable and so $A$ must be a permutation. 
We show in the next section that there is a class of matrices such that some groups are not in $B_{X}(R)$ for any $R$ in this class.

The question now arises, "Do we always have either all groups or only trivial groups?" This is answered negatively in the next section.

3. $B_{X}(R)$ containing only some groups. In this section we look at a class of matrices for which some, but not all, groups appear in $B_{X}(R)$ for $R$ in this class. We show that for any $R$ in this class the maximal groups in $B_{X}(R)$ are a special type.

Consider the class $\Gamma$ of matrices having the block form

$$
\left(\begin{array}{ll}
I_{k} & A \\
0 & 0
\end{array}\right)
$$

where $I_{k}$ is the $k \times k$ identity matrix and $A$ is a $k \times n$ matrix whose $(1,1)$ entry is a 1 and all other entries are 0 . We will establish our results for matrices in this class and show the results also hold for matrices of the forms

$$
\left(\begin{array}{ll}
I_{k} & A \\
0 & 0
\end{array}\right) \quad \text { and } \quad\left(\begin{array}{cc}
I_{k} & 0 \\
A & 0
\end{array}\right)
$$

where $A$ has exactly one nonzero entry. Throughout this section all sandwich matrices $R$ will be in $\Gamma$.

THEOREM 4. The following are necessary and sufficient for $E$ to be an $R$-idempotent.

(i) Assume row $j$ has a 1 in the $(j, 1)$ position. If row $j$ also has a 1 in positions $P_{1}, \cdots, P_{m}$, then row $j$ is the sum of rows 1 , $k+1$ and rows $P_{1}, \cdots, P_{m}$. Otherwise it is just the sum of rows 1 and $k+1$.

(ii) Assume row $j$ has a 0 in the $(j, 1)$ position. If row $j$ also has $a 1$ in positions $P_{1}, \cdots, P_{m}$, then row $j$ is the sum of rows $P_{1}, \cdots, P_{m}$. If there are no such rows $p_{i}$, then row $j$ is zero.

Proof. Let $E R E=E$. Since rows $k+1$ through $n$ of $R$ are zero, then columns $k+1$ through $n$ of $E$ do not affect the product $E R$. Thus, we consider entries in columns 1 through $k$ of $E$.

(i) If row $j$ has a 1 in the $(j, 1)$ position, then $\left\{x_{1}, x_{k+1}\right\}$ is in $x_{j} E R$. Thus $\left\{x_{1}, x_{k+1}\right\} E$ is in $x_{j} E R E=x_{j} E$ and rows 1 nad $k+1$ are in row $j$. That is, row $j$ has 1 's at least where rows 1 and $k+1$ have 1's. If row $j$ has a 1 in the $\left(j, p_{i}\right)$ position for $p_{i}$ in $\{2, \cdots, k\}$, then $x_{p_{i}}$ is in $x_{j} E R$ and $x E_{p_{i}}$ is in $x_{j} E R E=x_{j} E$ and row $p_{i}$ is contained in row $j$. Clearly if the $\left(j, p_{i}\right)$ entry is 0 , then $x_{p_{i}}$ is not in 
$x_{j} E R$ and hence row $p_{i}$ is not in $x_{j} E R E=x_{j} E$. Thus, $x_{j} E=x_{j} E R E=$ $\left\{x_{1}, x_{p_{1}}, \cdots, x_{p_{m}}, x_{k+1}\right\} E$ where the $\left(j, p_{i}\right)$ entries are nonzero, and the result follows.

(ii) From the proof of (i) we see $x_{j} E=x_{j} E R E=\left\{x_{p_{1}}, \cdots, x_{p_{m}}\right\} E$ where the $\left(j, p_{i}\right)$ entry is a 1 , and the result follows.

Conversely, consider row $j$ of $E$. We show $x_{j} E=x_{j} E R E$. If row $j$ has a 1 in the $(j, 1)$ position and in the $\left(j, p_{i}\right), \cdots,\left(j, p_{m}\right)$ positions for $p_{t}$ in $\{2, \cdots, k\}$, then $x_{j} E R E=\left\{x_{1}, x_{p_{1}}, \cdots, x_{p_{m}}, x_{k+1}\right\} R E=$ $\left\{x_{1}, x_{p_{1}}, \cdots, x_{p_{m}}, x_{k+1}\right\} E$. By hypothesis, row $j$ is the sum of rows 1 , $p_{1}, \cdots, p_{m}, k+1$ and $x_{j} E=\left\{x_{1}, x_{p_{1}}, \cdots, x_{p_{m}}, x_{k+1}\right\} E$. If row $j$ has a 0 in the $(j, 1)$ position, then the proof is similar except we exclude $x_{1}$ and $x_{k+1}$.

EXAMPLE 1. If $n=7$ and $k=4$, then the matrix

$$
E=\left(\begin{array}{lllllll}
1 & 1 & 0 & 0 & 1 & 0 & 0 \\
0 & 1 & 0 & 0 & 0 & 0 & 0 \\
0 & 1 & 1 & 0 & 0 & 0 & 0 \\
1 & 1 & 1 & 1 & 1 & 1 & 1 \\
1 & 1 & 0 & 0 & 1 & 0 & 0 \\
1 & 1 & 1 & 1 & 1 & 1 & 1 \\
0 & 0 & 0 & 0 & 0 & 0 & 0
\end{array}\right)
$$

is an $R$-idempotent, but the matrix

$$
F=\left(\begin{array}{lllllll}
1 & 1 & 0 & 0 & 1 & 0 & 0 \\
1 & 1 & 0 & 0 & 0 & 0 & 0 \\
0 & 1 & 1 & 0 & 0 & 0 & 0 \\
1 & 1 & 0 & 1 & 1 & 0 & 0 \\
1 & 1 & 1 & 1 & 1 & 0 & 0 \\
0 & 0 & 0 & 0 & 0 & 0 & 0 \\
0 & 0 & 0 & 0 & 0 & 0 & 0
\end{array}\right)
$$

is not an $R$-idempotent.

We now look at elements in $G_{E}(R)$.

THeOREM 5. Let $A$ be in $G_{E}(R)$.

(i) Row $m$ of $A$ is zero if and only if row $m$ of $E$ is zero.

(ii) Rows $j$ and $m$ of $A$ are equal if and only if rows $j$ and $m$ of $E$ are equal.

(iii) Row $m$ of $A$ is the sum of a subset of the rows 1 through $k+1$ of $E$.

(iv) Row $j$ of $A$ is the sum of rows $p_{1}, \cdots, p_{t}$ of $A$ if and only if row $j$ of $E$ is the sum of rows $p_{1}, \cdots, p_{t}$ of $E$. 
Proof. (i) and (ii) follow directly from $A R A^{\prime}=E$ and $E R A=A$ where $A^{\prime}$ denotes the $R$-inverse of $A$.

(iii) From $A R E=A$ we have row $m$ of $A$ is

$$
\left(\begin{array}{c}
a_{m 1} e_{11}+a_{m 2} e_{21}+\cdots+a_{m k} e_{k 1}+a_{m 1} e_{k+1,1} \\
a_{m 1} e_{12}+a_{m 2} e_{22}+\cdots+a_{m k} e_{k 2}+a_{m 1} e_{k+1,2} \\
\cdots \cdots \cdots \cdots \cdots \cdots \cdots \cdots \cdots \cdots \cdots \cdots \cdots \cdots \cdots \cdots \cdots \cdots \\
a_{m 1} e_{1 n}+a_{m 2} e_{2 n}+\cdots+a_{m k} e_{k n}+a_{m 1} e_{k+1, n}
\end{array}\right)
$$

where $a_{i j}\left(e_{i j}\right)$ is the $(i, j)$ entry of $A(E)$. If $a_{m p_{1}}, \cdots, a_{m p_{j}}=0$ and $a_{m p_{j+1}}, \cdots, a_{m p_{q}}=1$ where $p_{i}$ is in $\{1, \cdots, k\}$, then row $m$ of $A$ is

$$
\left(e_{p_{j+1}, 1}+e_{p_{j+2}, 1}+\cdots+e_{p_{q}, 1} \cdots e_{p_{j+1}, n}+e_{p_{j+2}, n}+\cdots+e_{p_{q}, n}\right)
$$

which is the sum of rows $p_{j+1}, \cdots, p_{q}$ of $E$. If $a_{m 1}=1$, then we also have $e_{k+1, t}$ in each entry where $t$ runs from 1 to $n$.

(iv) If row $j$ of $A$ is the sum of rows $p_{1}, \cdots, p_{t}$ of $A$ and if $A^{\prime}$ denotes the $R$-inverse of $A$ we have

$$
x_{j} E=x_{j} A R A^{\prime}=\left\{x_{p_{1}}, \cdots, x_{p_{t}}\right\} A R A^{\prime}=\left\{x_{p_{1}}, \cdots, x_{p_{t}}\right\} E .
$$

The converse is similar.

Thus, for example, if $X$ has 7 elements and $k=4$ and row $m$ of $A$ is ( $\left.\begin{array}{lllllll}1 & 0 & 1 & 1 & 0 & 0 & 1\end{array}\right)$, then this row is the sum of rows $1,3,4$ and 5 of $E$.

We remark here that this theorem is also valid if $R$ has the form

$$
\left(\begin{array}{cc}
I_{k} & A \\
0 & 0
\end{array}\right)
$$

where $A$ has exactly one nonzero entry, say the $(i, j)$ entry where $j \geqq k+1$ is nonzero. For in the above proof we use row $i$ where we previously used row 1 and column $j$ where we used column $k+1$. Similarly, by using the word "column" where we used "row" the result also holds for any $R$ of the form

$$
\left(\begin{array}{ll}
I_{k} & 0 \\
A & 0
\end{array}\right)
$$

where $A$ has exactly one nonzero entry.

The goal now is to show how to construct an arbitrary $A$ in $G_{E}(R)$ and thereby show only certain groups arise in $B_{X}(R)$. From Theorems 4 and 5 (iv) we see that we need only show the construction of the first $k+1$ rows of $A$. The remaining rows are determined by their pattern in $E$. That is, if row $m$ of $E$, for $m>k+1$, is the of rows $p_{1}, \cdots, p_{t}$ or $E$ where $p_{i}$ is between $q$ and $k+1$ inclusive, then row $m$ of $A$ is the sum of rows $p_{1}, \cdots, p_{t}$ of $A$. We make the following definitions which are illustrated in Example 2. 
DEFinition 1. Let $S$ be a sum of a subset of the first $k+1$ rows of $A$, but $S$ is not one of the first $k+1$ rows of $A$ (and may not even be any row of $A$ ). Then $S$ is called a row associated with $A$. If any row of $A$ or row associated with $A$ is the sum of rows $p_{1}, \cdots, p_{t}$, then each $p_{i}$ is called a summand. $S$ is the maximal sum of rows $p_{1}, \cdots, p_{t}$ if every one of the first $k+1$ rows contained in $A$ is a $p_{i}$. We also refer to $S$ as a maximal row associated with $A$.

Definition 2. Each row $m$ of $A$ is the sum of a subset of the first $k+1$ rows of $A$ and some of the associated rows of $A$. Let row $m$ be listed as a summand only if it is not the sum of rows distinct (not necessarily different) from itself. Then we say the sum is maximal if all rows contained in row $m$ and all maximal rows associated with $A$ contained in row $m$ are listed as summands. If row $m$ is the maximal sum of $N$ rows we write $S_{m}(A)=N$ and say row $m$ has order $N$.

When we say row $m$ of $A$ is a sum of $N$ rows of $A$, we mean each summand is either one of the first $k+1$ rows of $A$ or a row associated with $A$.

We now make the following classification of the nonzero rows of $A$ and the rows associated with $A$.

DeFinition 3. If every summand of row $m$ is identical to row $m$, then row $m$ is called an independent row. If at least one summand of row $m$ is proper and if row $m$ is not the sum of its proper summands, then it is called fixed. If at least one summand of row $m$ is proper and if row $m$ is the sum of its proper summands, then it is called dependent.

By this definition rows associated with $A$ are dependent. Thus, when we refer to a dependent row, it may or may not be in $A$.

Example 2. Let $A$ be given below where $k=8$.

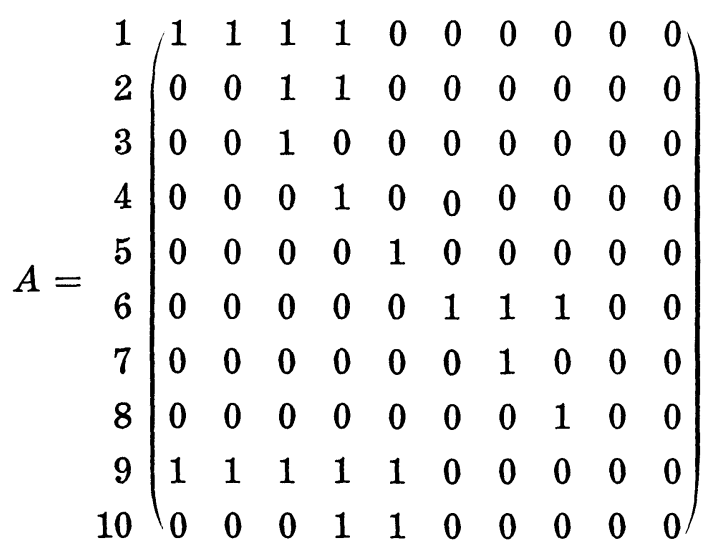


$S_{i}(A)=1$ for $i=3,4,5,7$ and 8 and $S_{1}(A)=4$ (sum of rows $1,2,3$ and 4 ), $S_{2}(A)=2$ (sum of rows 3 and 4 ) and $S_{10}(A)=2$ (sum of rows 4 and 5). We also have row 6 is the sum of rows 6,7 and 8 and $S$ where $S$ is the sum of rows 7 and 8 and so $S_{6}(A)=4$. Row 9 is the sum of rows 1 through 5 and $S_{1}, S_{2}$ and $S_{3}$ where $S_{1}$ is the sum of rows 3 and $5, S_{2}$ is the sum of rows 4 and 5 and $S_{3}$ is the sum of

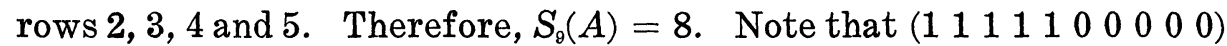
considered as the sum of rows 1 and 5 of $A$ is associated with $A$, but would not be a maximal row associated with $A$ unless we considered it as the sum of rows $1,2,3,4,5$ and 9 of $A$. Rows 3,4 , 5,7 and 8 are independent, rows 1,6 and 9 are fixed, and rows 2 and 10 are dependent.

The following sequence of propositions will enable us to construct an arbitrary element in $G_{E}(R)$ for an $R$-idempotent $E$. Throughout we let $A$ be in $G_{E}(R)$.

\section{Proposition 1.}

(i) Row $m$ of $E$ is independent if and only if row $m$ of $A$ is independent.

(ii) Row $m$ of $E$ is fixed if and only if row $m$ of $A$ is fixed.

(iii) Row $m$ of $E$ is dependent if and only if row $m$ of $A$ is dependent.

Proof. We prove the "if" part of (i), (ii) and (iii) and the "only if" parts must follow.

(i) Let row $m$ of $E$ be the maximal sum of rows $p_{1}, \cdots, p_{t}$ of $E$. Each of these rows will be identical to row $m$. Thus, by Theorem 6 (ii) and (iv) row $m$ of $A$ is the maximal sum of rows $p_{1}, \cdots, p_{t}$ all just like row $m$ of $A$ and row $m$ of $A$ is independent.

(ii) Let row $m$ of $E$ be the maximal sum of rows $p_{1}, \cdots, p_{t}$ where either $m$ is a $p_{i}$ or some row $p_{i}$ is identical to row $m$. Apply Theorem 6 (ii) and (iv) to show row $m$ of $A$ is the maximal sum of rows $p_{1}, \cdots, p_{t}$ of $A$ where either $m$ is a $p_{i}$ or some row $p_{i}$ is identical to row $m$. Thus, row $m$ of $A$ is fixed.

(iii) As above, apply the definition of dependent row along with Theorem 6 (ii) and (iv).

Proposition 2. $S_{m}(E)=N$ if and only if $S_{m}(A)=N$.

Proof. Assume $A \neq E$ or there is nothing to prove. Assume $S_{m}(E)=N$ and row $m$ of $E$ is the maximal sum of rows $p_{1}, \cdots, p_{N}$ of $E$. Assume rows $p_{1}, \cdots, p_{j}$ are in $E$ (as usual $p_{i}$ is between 1 and $k+1$ inclusive) and rows $p_{j+1}, \cdots, p_{N}$ are maximal associated 
with $E$. Thus, row $m$ of $E$ is the sum of rows $p_{1}, \cdots, p_{j}$ of $E$ (not maximal unless $j=N$ ), and so row $m$ of $A$ is the sum of rows $p_{1}, \cdots, p_{j}$ of $A$.

Assume row $p_{q}$ is one of the dependent rows associated with $E$ and is the sum of rows $p_{z_{1}}, \cdots, p_{z_{t}}$ of $E$ where $p_{z_{i}}$ is between 1 and $j$ inclusive. Then the sum of rows $p_{z_{1}}, \cdots, p_{z_{t}}$ of $A$ is associated with $A$. For if it were one of the first $k+1$ rows of $A$, say row $q$, then by Theorem 6 (ii) row $q$ of $E$ would be the sum of rows $p_{z_{1}}, \cdots, p_{z_{t}}$ of $E$. But this sum is not a row of $E$. Similarly, for each row $p_{t}$ associated with $E$, we get a corresponding row $p_{t}$ associated with $A$. Furthermore, each is maximal in $A$ since it was in $E$. Thus $S_{m}(A)$ is greater than or equal to $N$. If $S_{m}(A)$ is strictly greater than $N$, then either there is another row in $A$ in the sum of row $m$ or another row associated with $A$ in the sum. In the former case, we contradict Theorem 5 (ii), in the latter case this associated row of $A$ will give rise to another associated row of $E$ contradicting the fact that the sum was maximal.

Conversely assume $S_{m}(A)=N$ and $S_{m}(E)=M \neq N$. But by the above $S_{m}(E)=M$ implies $S_{m}(A)=M$ and we have a contradiction.

Proposition 3. Given the fixed and independent rows of $A$ we can determine the dependent rows of $A$.

Proof. The dependent rows of $A$ will be in the same positions as the dependent rows of $E$. Let row $m$ of $E$ be dependent and the maximal sum of rows $p_{1}, \cdots, p_{t}$ of $E$ where rows $p_{1}, \cdots, p_{j}$ are dependent. By the definition of maximal sum, every summand of any row $p_{i}$ for $i$ between 1 and $j$ inclusive will be one of the rows $p_{1}, \cdots, p_{t}$ and by the definition of dependent row, each summand is proper. Thus, dependent rows are redundant in a maximal sum, and row $m$ of $E$ is the sum of rows $p_{j+1}, \cdots, p_{t}$ of $E$ where each $p_{i}$ is independent or fixed. By Theorem 5 (ii) and Proposition 3 row $m$ of $A$ is the sum of rows $p_{j+1}, \cdots, p_{t}$ of $A$ which will be fixed or independent as they are in $E$.

From Theorem 5 (ii) and Propositions 1 and 2 we have the following proposition.

Proposition 4. Row $m$ of $A$ has the same unmber and types of summands as row $m$ of $E$.

Proposition 4 is useful in constructing the independent and fixed rows of $A$. Recall, each independent row of $E$ is a row of $E$. That is, it cannot be associated with $E$. By Theorem 5 (ii) and Proposi- 
tion 1 each of these rows must be an independent row of $A$. Similarly, each fixed row of $E$ must be some fixed row of $A$. The following definitions help us apply Proposition 4.

DEFINITION 4. If an independent row is a summand of a fixed row, it is called Type 1 . Otherwise it is Type 2.

Propositions 1 and 4 now give the following.

Proposition 5. Row $m$ of $E$ is independent of Type 1 (Type 2) if and only if row $m$ of $A$ is independent of Type 1 (Type 2).

Definition 5. A fixed row of $A$ is called a maximal fixed row (MFR) if it is not the summand of any fixed row different from itself. An MFR together with its summands is called a maximal fixed block (MFB). MFRs (or MFBs) with the same number and types of summands are said to be in the same class. We define a sub-MFR (sub-MFB) to be any MFR (MFB) within an MFR (MFB). A fixed row is a minimal fixed row (mFR) if it does not contain any fixed summands. An mFR together with its summands is called a minimal fixed block (mFB).

We remark that a fixed row may be both an MFR and an mFR. Every MFB is either an $\mathrm{mFB}$ or contains an $\mathrm{mFB}$.

EXAMPLE 3. Let

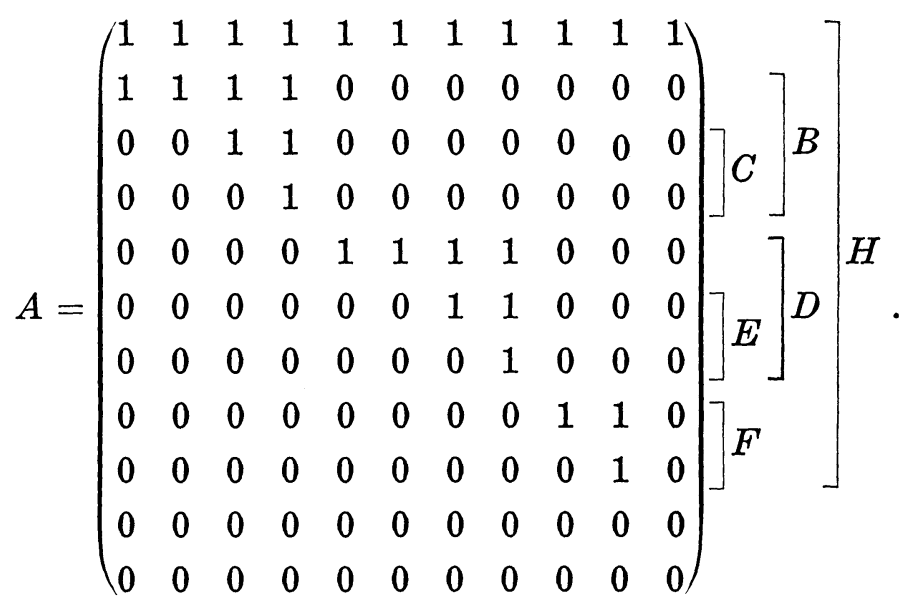

$H$ is an MFB witn $B, D$ and $F$ as sub-MFBs. $B$ and $D$ are in the same class. $C$ and $E$ are sub-MFBs of $B$ and $D$ respectively and are mFBs. $F$ is also an $\mathrm{mFB}$.

Proposition 4 now gives us the following: 
Proposition 6. Row $m$ of $E$ is an MFR with an associated MFB in class $\Gamma$ if and only if row $m$ of $A$ is an MFR with associated MFB in class $\Gamma$.

We now give the construction of the first $k+1$ rows of $A$.

Step 1. If any rows of $E$ are zero, then the corresponding rows in $A$ are zero.

Step 2. Distict independent rows of Type 2 in $E$ are permuted observing Theorem 5 (ii).

Step 3. MFBs of the same class in $E$ are permuted to form MFBs of this class in $A$. We must observe Propositions 1 and 2. That is, subblocks may need to be permuted within an MFB.

Step 4. If within an MFB there are independent rows of Type 2 (thus, they are actually independent rows of Type 1 in $E$ ), then they may be permuted.

Step 5. Repeat Steps 3 and 4 with sub-MFBs. That is, subMFBs of the same MFB and of the same class may be permuted and within them, independent rows of Type 2 may be permuted.

Step 6. Repeat Step 4 until $\mathrm{mFBs}$ have been permuted and their independent rows of Type 2 have been permuted.

Step 7. Calculate the dependent rows by the fixed and independent rows and the pattern of $E$ (as in the proof of Proposition 3 ).

THeOREM 6. $A$ is in $G_{E}(R)$ if and only if $A$ is constructed as above.

Proof. If $A$ is in $G_{E}(R)$, then Propositions 1 through 6 show that is $A$ constructed as above. Conversely, let $A$ be constructed as above. We must show $A * E=A=E * A$ and the existence of an inverse. We first show $A * E=E * A=A$.

Case 1. Row $m$ of $A$ is independent or fixed. Then it is some row of $E$, say row $p$. Thus, $x_{m} A=x_{p} E$ and $x_{m} A * E=x_{p} E * E=$ $x_{p} E=x_{m} A$. Assume row $m$ of $E$ has ones in the $p_{1}, \cdots, p_{t}$ positions for $p_{i}$ between 1 and $k$ inclusive. Row $m$ is the sum of rows $p_{1}, \cdots, p_{t}$ if the $(m, 1)$ position is a zero and so $x_{m} E=x_{m} E R$. It is the sum of rows $p_{1}, \cdots, p_{t}, k+1$ if the $(m, 1)$ position is a 1 . In 
the former case, row $m$ of $A$ is the sum of rows $p_{1}, \cdots, p_{t}$ of $A$ and $x_{m} E * A=x_{m} E A=\left\{x_{p_{1}}, \cdots, x_{p_{t}}\right\} A=x_{m} A$. In the latter case row $m$ of $A$ is the sum of rows $p_{1}, \cdots, p_{t}, k+1$ of $A$ and $x_{m} E R A=$ $\left\{x_{p_{1}}, \cdots, x_{p_{t}}, x_{k+1}\right\} R A=\left\{x_{p_{1}}, \cdots, x_{p_{t}}, x_{k+1}\right\} A=x_{m} A$.

Case 2. Row $m$ of $A$ is dependent. Then row $m$ of $E$ is dependent. Assume row $m$ of $E$ is the sum of rows $p_{1}, \cdots, p_{t}$ of $E$ where row $p_{i}$ is fixed or independent. Thus, row $m$ of $A$ is the sum of rows $p_{1}, \cdots, p_{t}$ of $A$ where row $p_{i}$ is fixed or inedpendent in $A$. Thus, from Case 1 , for each $p_{i}$ we have $x_{p_{i}} A * E=x_{p_{i}} A=x_{p_{i}} E * A$. Now, $x_{m} A * E=\left\{x_{p_{1}}, \cdots, x_{p_{t}}\right\} A * E=x_{p_{1}} A * E+x_{p_{2}} A * E+\cdots+x_{p_{t}} A * E=$ $x_{p_{1}} A+x_{p_{2}} A+\cdots+x_{p_{t}} A=\left\{x_{p_{1}}, \cdots, x_{p_{i}}\right\} A=x_{m} A$. Similarly $x_{m} E * A=$ $x_{m} A$.

We now construct a $B$ by the above rules and show $B$ is an $R$-inverse of $A$.

Step 1. If row $m$ of $E$ is zero, then row $m$ of $B$ is zero.

Step 2. Independent rows of Type 2. Assume rows $p_{1}, \cdots, p_{t}$ of $E$ are distinct independent rows of Type 2. Let $\theta$ be the permutation on $p_{1}, \cdots, p_{t}$ where row $p_{i}$ of $E$ is row $\theta\left(p_{i}\right)$ of $A$. Let these independent rows be permuted in $B$ by $\theta^{-1}$. That is, row $\theta\left(p_{i}\right)$ of $E$ is row $p_{i}$ of $B$.

Step 3. MFBs of the same class. Permute these in $B$ following the same scheme above for independent rows of Type 2 .

Step 4. Independent rows of Type 2 within an MFB. Let MFBs $B_{1}, \cdots, B_{t}$ be of the same class and let each $B_{i}$ have distinct independent rows $b_{i 1}, b_{i 2}, \cdots, b_{i t}$ of Type 2 . Assume $\theta$ permutes the blocks as they are permuted in $A$ (similar to $\theta$ in Step 2). Then in $A$, block $B_{i}$ occupies the position $\theta\left(B_{i}\right)$ occupies in $E$ and in $B$, block $\theta\left(B_{i}\right)$ occupies the position block $B_{i}$ does in $E$. If rows $b_{i 1}, \cdots, b_{i t}$ of block $B_{i}$ have been permuted in $A$, then apply the same permutation to the corresponding rows in block $\theta\left(B_{i}\right)$ of $B$.

Step 5. Sub-MFRs. These are formed in $B$ following the same scheme as for independent rows in Step 4.

Step 6. Continue as in Steps 4 and 5 for independent rows of Type 2 within sub-MFBs and for sub-MFBs within the sub-MFBs until the process terminates with mFBs.

Step 7. Dependent rows. These are determined by independent and fixed rows. 
Thus we have a $B$ such that $B * E=B=E * B$. Let the independent rows of Type 2 in $A$ and $B$ be as in Step 2 above. Then for each $i, x_{\theta\left(p_{i}\right)}(A * B)=x_{p_{i}}(E * B)=x_{p_{i}}(B)=x_{\theta\left(p_{i}\right)}(E)$. Similarly for each $i, \quad x_{p_{i}}(B * A)=x_{\theta\left(p_{i}\right)}(E * A)=x_{\theta\left(p_{i}\right)}(A)=x_{p_{i}}(E)$. Thus, for any independent row, say $x_{m}$, of Type 2 we have $x_{m}(A * B)=x_{m} E=$ $x_{m}(B * A)$. Similar proofs give the same result for MFRs. Now consider independent rows of Type 2 within an MFB as in Step 4. By the construction, if row $m$ of $E$ is row $p$ of $A$, then row $p$ of $E$ is row $m$ of $B$ where row $m$ is in $B_{i}$ and row $p$ is in $\theta\left(B_{i}\right)$. This implies $x_{m}(E)=x_{p}(A)$ and $x_{p}(E)=x_{m}(B)$ and for each row $m$ in $B_{i}$ we have $x_{m}(E)=x_{p}(A)=x_{p}(E * A)=x_{m}(B * A)$. Similarly, if row $m$ of $E$ is row $q$ of $B$, then row $q$ of $E$ is row $m$ of $A$ and $x_{m}(E)=$ $x_{q}(B)=x_{q}(E * B)=x_{m}(A * B)$. Thus, for these rows $x_{m}(A * B)=x_{m} E=$ $x_{m}(B * A)$. Sub-MFRs satisfy $x_{m}(A * B)=x_{m} E=x_{m}(B * A)$ by the same type of proof. We now show the result for dependent rows. Let row $m$ of $E$ be dependent. Then it is the sum of rows $p_{1}, \cdots, p_{t}$ of $E$ which are fixed or independent, and rows $m$ of $A$ and $B$ are the sums of rows $p_{1}, \cdots, p_{t}$ of $A$ and $B$ respectively. Since $x_{m}(A * B)=x_{m} E=x_{m}(B * A)$ for row $x_{m}$ fixed or independent, we have $x_{m} E=\left\{x_{p_{1}}, \cdots, x_{p_{t}}\right\} E=\left\{x_{p_{1}}\right\} E+\cdots+\left\{x_{p_{t}}\right\} E=\left\{x_{p_{1}}\right\} A * B+\cdots+$ $\left\{x_{p_{t}}\right\} A * B=\left\{x_{p_{1}}, \cdots, x_{p_{t}}\right\} A * B=x_{m}(A * B)$. Similarly, $x_{m} E=x_{m}(B * A)$.

Corollary 1. $C_{E}(R)$ is trivial if and only if

(i) No two distinct independent rows of Type 2 are in $E$.

(ii) No independent rows of Type 1 can be permuted.

(iii) No two fixed rows of $E$ are in the same class.

CoRollary 2. $G_{E}(R)$ is nontrivial if and only if it contains a nontrivial subgroup isomorphic to a permutation group.

Proof. Assume $G_{E}(R)$ is nontrivial. Then at least one of the three statements of Corollary 1 must be false. Assume (i) is false and let $p_{1}, \cdots, p_{t}$ be the distrinct independent rows of Type 2 . Let $A$ be the set of all $A$ in $G_{E}(R)$ formed by permuting rows $p_{1}, \cdots, p_{t}$ of $E$ and leaving all other rows of $E$ stationary. $A$ is a subgroup of $G_{E}(R)$ isomorphic to the permutation group on $\left\{p_{1}, \cdots, p_{t}\right\}$. A similar proof establishes the result if we assume (ii) or (iii) is false.

The converse is clear.

If for each $N_{i}$ in $\left\{N_{1}, \cdots, N_{p}\right\}$ there are $n_{i}$ identical independent rows of Type 2 and also if for each $C_{k}$ in the set $\left\{C_{1}, \cdots, C_{j}\right\}$ there are $c_{k}$ MFBs of class $C_{k}$ where $c_{k}$ is greater than 1 , then $G_{E}(R)$ contains a subgroup isomorphic to $G=P_{p} \times P_{C_{1}} \times P_{C_{2}} \times \cdots \times P_{C_{t}}$ where $P_{T}$ is the permutation group on the set of $T$ elements. As in the proof of Theorem 6 let $\mathscr{A}$ in $G_{E}(R)$ be the set of all $A$ such 
that the independent rows of Type 1 are fixed. Then $A \simeq G$. Thus we have the following

CoRollary 3. If $E$ contains no independent rows of Type 1 that can be permuted or if no MFBs are of the same class, then $G_{E}(R)$ is isomorphic to a direct product of permutation groups.

EXAMPLE 4. Let $k=6$ and

$$
E=\left(\begin{array}{llllllll}
1 & 0 & 0 & 0 & 0 & 0 & 0 & 0 \\
0 & 1 & 1 & 0 & 0 & 0 & 0 & 0 \\
0 & 1 & 1 & 0 & 0 & 0 & 0 & 0 \\
0 & 0 & 0 & 1 & 1 & 1 & 0 & 0 \\
0 & 0 & 0 & 0 & 1 & 1 & 0 & 0 \\
0 & 0 & 0 & 0 & 1 & 1 & 0 & 0 \\
1 & 0 & 0 & 0 & 0 & 0 & 0 & 0 \\
1 & 0 & 0 & 0 & 0 & 0 & 0 & 0
\end{array}\right) .
$$

Rows $1,2,3,7$ and 8 are independent of Type 2; but since rows 1,7 and 8 are alike and 2 and 3 are alike, we only get one permutation from these. Row 4 is fixed and rows 5 and 6 are independent of Type 1. Thus, $G_{E}(R)=\{E, A\}$ where

$$
A=\left(\begin{array}{llllllll}
0 & 1 & 1 & 0 & 0 & 0 & 0 & 0 \\
1 & 0 & 0 & 0 & 0 & 0 & 0 & 0 \\
1 & 0 & 0 & 0 & 0 & 0 & 0 & 0 \\
0 & 0 & 0 & 1 & 1 & 1 & 0 & 0 \\
0 & 0 & 0 & 0 & 1 & 1 & 0 & 0 \\
0 & 0 & 0 & 0 & 1 & 1 & 0 & 0 \\
0 & 1 & 1 & 0 & 0 & 0 & 0 & 0 \\
0 & 1 & 1 & 0 & 0 & 0 & 0 & 0
\end{array}\right) .
$$

EXAMPLE 5. Let $k=8$ and

$$
E=\left(\begin{array}{llllllllll}
1 & 0 & 0 & 0 & 0 & 0 & 0 & 0 & 0 & 0 \\
0 & 1 & 1 & 1 & 0 & 0 & 0 & 0 & 0 & 0 \\
0 & 0 & 1 & 1 & 0 & 0 & 0 & 0 & 0 & 0 \\
0 & 0 & 1 & 1 & 0 & 0 & 0 & 0 & 0 & 0 \\
0 & 0 & 0 & 0 & 1 & 1 & 1 & 1 & 0 & 0 \\
0 & 0 & 0 & 0 & 0 & 1 & 1 & 1 & 0 & 0 \\
0 & 0 & 0 & 0 & 0 & 0 & 1 & 1 & 0 & 0 \\
0 & 0 & 0 & 0 & 0 & 0 & 0 & 1 & 0 & 0 \\
0 & 0 & 0 & 0 & 0 & 0 & 0 & 1 & 0 & 0 \\
1 & 0 & 0 & 0 & 0 & 0 & 0 & 0 & 0 & 0
\end{array}\right) .
$$


Row 1 is independent of Type 2. Row 2 is an MFR with rows 2, 3 and 4 as summands and so $S_{2}(E)=3$. Row 5 is an MFR with rows $5,6,7,8$ and 9 as summands and so $S_{5}(E)=5$. Rows 6 through 9 form a sub-MFB of row 5. From the above we see no permutations can be formed and $G_{E}(R)$ is trivial.

ExAMPle 6 . Let $k=16$ and $R$ be $18 \times 18$. Let

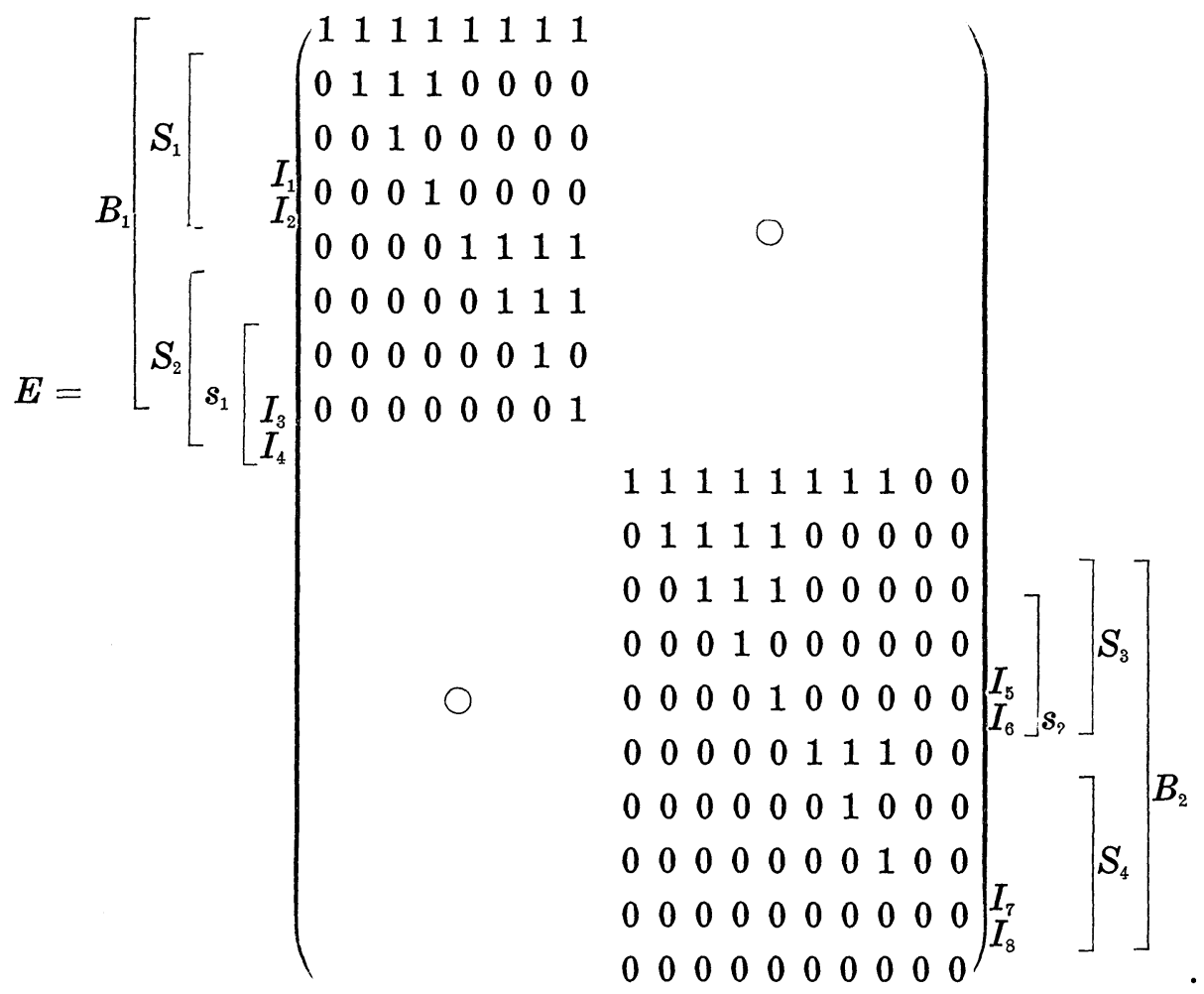

$B_{1}$ and $B_{2}$ are MFBs of the same class and can be permuted. $S_{1}$ and $S_{2}$ are sub-MFBs of $B_{1}, s_{1}$ is a sub-MFB of $S_{2}$. Similarly, $S_{3}$ and $S_{4}$ are sub-MFBs of $B_{2}$ and $s_{2}$ is a sub-MFB of $S_{3}$. Note $s_{1}$ and $s_{2}$ are mFBs and $I_{1}$ through $I_{8}$ are independent of Type $1 . S_{1}$ and $S_{2}$ (and $S_{3}$ and $\left.S_{4}\right)$ are not of the same class. The pairs $\left(I_{1}, I_{2}\right),\left(I_{3}\right.$, $\left.I_{4}\right),\left(I_{5}, I_{6}\right)$ and $\left(I_{7}, I_{8}\right)$ are independent of Type 2 within blocks $S_{1}$, $s_{1}, s_{2}$ and $S_{4}$ respectively and can be permuted within these blocks. Observe, if we permute $B_{1}$ and $B_{2}$, then we must permute $S_{1}$ and $S_{2}$ and $S_{3}$ and $S_{4}$ within the blocks. Thus we can describe $G_{E}(R)$ as follows. If we do not permute $B_{1}$ and $B_{2}$, then we have 16 elements of this form-one for each of the possible permutations of the pairs of independent rows. If we do permute $B_{1}$ and $B_{2}$, then we again have 16 elements. Thus, $G_{E}(R)$ has 32 elements. The first 16 elements described form the subgroup $K=S_{2} \times S_{2} \times S_{2} \times S_{2}$ 
where $S_{2}$ is the symmetric group on the set of two elements. For example the element

$$
\left(\left(\begin{array}{ll}
1 & 2 \\
1 & 2
\end{array}\right),\left(\begin{array}{ll}
1 & 2 \\
2 & 1
\end{array}\right),\left(\begin{array}{ll}
1 & 2 \\
2 & 1
\end{array}\right),\left(\begin{array}{ll}
1 & 2 \\
1 & 2
\end{array}\right)\right)
$$

in $K$ corresponds to the element $A$ in $G_{E}(R)$ with rows $I_{3}$ and $I_{4}$ and $I_{5}$ and $I_{6}$ interchanged. Rows $I_{1}, I_{2}, I_{7}$ and $I_{8}$ are not permuted. We can consider elements of $G_{E}(R)$ as 5 -tuples $(A, B, C, D, E)$ where each entry is a permutation of 1,2 . $A$ represents the permutation of $B_{1}$ and $B_{2}, B, C, D$ and $E$ represent the permutations of the pairs $\left(I_{1}, I_{2}\right),\left(I_{3}, I_{4}\right),\left(I_{5}, I_{6}\right)$ and $\left(I_{7}, I_{8}\right)$ respectively. Consider the elements where $A$ is the identity to be of Type 1 , and those where $A$ represents the permutation of $B_{1}$ and $B_{2}$ to be of Type 2. Let $X=(A, B, C, D, E)$ and $Y=\left(A^{\prime}, B^{\prime}, C^{\prime}, D^{\prime}, E^{\prime}\right)$ be elements of $G_{E}(R)$. The multiplication in $G_{E}(R)$ is given by

$$
X Y=\left\{\begin{array}{l}
\left(A A^{\prime}, B B^{\prime}, C C^{\prime}, D D^{\prime}, E E^{\prime}\right) \text { if } X \text { and } Y \text { are both Type } 1 \\
\left(A A^{\prime}, B E^{\prime}, C D^{\prime}, D C^{\prime}, E B^{\prime}\right) \text { if either } X \text { or } Y \text { is Type } 2 .
\end{array}\right.
$$

We remark that the above theorems and propositions are also valid if $R$ has the form

$$
\left(\begin{array}{ll}
I_{k} & A \\
0 & 0
\end{array}\right) \text { or }\left(\begin{array}{cc}
I_{k} & 0 \\
A & 0
\end{array}\right)
$$

where $A$ has exactly one nonzero entry. The proofs would be as indicated in the remarks following Theorem 5.

It is not known if there is a way to determine the maximal groups in $B_{X}(R)$ for any given $R$. It would be interesting to find properties of the relation $R$ that determine the maximal groups.

\section{REFERENCES}

1. K. Chase, New semigroups of binary relations, Semigroup Forum, 18 (1979), 79-82. 2. - Sandwich semigroups of binary relations, Discrete Math., 28 (1979), 231-236.

3. - Digraphs, automata and sandwich semigroups of binary relations, $\mathrm{Ph} . \mathrm{D}$. Thesis, Texas A \& M University, College Station, Texas (1978).

4. A. H. Clifford and G. B. Preston, The algebraic theory of semigroups, Vol. 1, Amer. Math. Soc., Providence, RI, 1961.

5. S. I. Montague and R. J. Plemmons, Maximal subgroups of the semigroup of relations, J. Algbra, 13 (1969), 575-587.

Received August 25, 1980.

The Pennsylvania State University

Department of Computer Sciences

UNIVERSITY PARK, PA 16802 



\section{PACIFIC JOURNAL OF MATHEMATICS}

\section{EDITORS}

DONALD BABBITT (Managing Editor)

University of California

Los Angeles, California 90024

Hugo RossI

University of Utah

Salt Lake City, UT 84112

C. C. MOORE and ARTHUR Agus

University of California

Berkeley, CA 94720

\section{J. DUGUNDJI}

Department of Mathematics University of Southern California Los Angeles, California 90007

R. FinN and J. MILGRAM Stanford University Stanford, California 94305

\section{ASSOCIATE EDITORS}
R. ARNES
E. F. BeCKENBACH
B. H. Neumann
F. WOLF
K. YosHIDA

\section{SUPPORTING INSTITUTIONS}

UNIVERSITY OF ARIZONA

UNIVERSITY OF BRITISH COLUMBIA

CALIFORNIA INSTITUTE OF TECHNOLOGY

UNIVERSITY OF CALIFORNIA

MONTANA STATE UNIVERSITY

UNIVERSITY OF NEVADA, RENO

NEW MEXICO STATE UNIVERSITY

OREGON STATE UNIVERSITY
UNIVERSITY OF OREGON UNIVERSITY OF SOUTHERN CALIFORNIA STANFORD UNIVERSITY UNIVERSITY OF HAWAII UNIVERSITY OF TOKYO UNIVERSITY OF UTAH WASHINGTON STATE UNIVERSITY UNIVERSITY OF WASHINGTON 


\section{Pacific Journal of Mathematics}

Vol. 100, No. $1 \quad$ September, 1982

Charalambos D. Aliprantis, Owen Sidney Burkinshaw and M. Duhoux,

Compactness properties of abstract kernel operators $\ldots \ldots \ldots \ldots \ldots \ldots 1$

Roger C. Alperin, Locally compact groups acting on trees .............23

Robert F. Brown, Real homology of Lie group homomorphisms ......... 33

Karen Chase, Maximal groups in sandwich semigroups of binary relations . . 43

W. Wistar (William) Comfort and T. Soundararajan, Pseudocompact

group topologies and totally dense subgroups $\ldots \ldots \ldots \ldots \ldots \ldots \ldots 61$

M. Ferri and C. Gagliardi, Crystallisation moves $\ldots \ldots \ldots \ldots \ldots \ldots \ldots$

Kenneth R. Goodearl, Directly finite aleph-nought-continuous regular

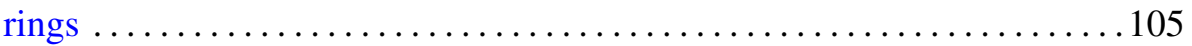

Edward Lewis Green, On the representation theory of rings in matrix

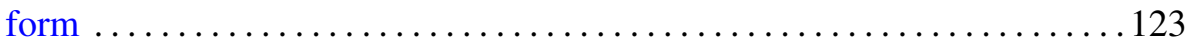

Walter Hengartner and Glenn E. Schober, Interpolation, continuation, and

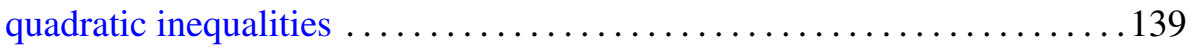

Kenneth Kunen and Haskell Paul Rosenthal, Martingale proofs of some geometrical results in Banach space theory $\ldots \ldots \ldots \ldots \ldots \ldots \ldots \ldots \ldots \ldots$

Brian William McEnnis, Shifts on indefinite inner product spaces. II . . . . . 177

Roman Pol, Note on the spaces $P(S)$ of regular probability measures whose topology is determined by countable subsets $\ldots \ldots \ldots \ldots \ldots \ldots \ldots \ldots 185$

Joan Manuel Verdera Melenchón, Finitely generated projective extensions

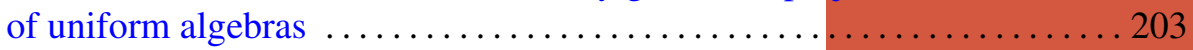

Cheng Ye You, Fixed point classes of a fiber map .................. 217 